\title{
Complications of Thyroidectomy in Hashimoto Thyroiditis vis-à-vis Benign Goitres
}

https://doi.org/10.47210/bjohns.2020.v28i3.317

Savita Lasrado, ${ }^{1}$ Anita Aramani, ${ }^{1}$ Aishwarya Hegde, ${ }^{1}$ Kuldeep Moras, ${ }^{1}$ Cimona Dsouza ${ }^{1}$

$\underline{\text { Introduction }}$

\section{ABSTRACT}

Surgery for Hashimoto thyroiditis (HT) can be difficult due to dense inflammatory process surrounding the gland and postoperatively patient can develop hypocalcaemia, recurrent laryngeal nerve injury, haematoma and infection. Purpose of this study was to compare the rate of post-operative complications in HT with that in benign goitres.

Materials and Methods

Data of the patients, who underwent thyroidectomy for benign goitres between January 2013 and December 2017, were reviewed retrospectively. Patients were divided in to two groups. Group A included patients confirmed to have Hashimoto thyroiditis $(n=148)$ and Group B included patients who underwent thyroidectomy for other benign thyroid disorders $(n=430)$.

$\underline{\text { Results }}$

A total of 578 patients who underwent thyroidectomy for benign thyroid diseases were included in the present retrospective study. Group A consisted of 148 patients who had HT. Group B consisted of 430 patients who had other benign thyroid diseases. The data was analysed using SPSS 23 and p-value < 0.05 was considered as statistically significant. Patients undergoing thyroidectomy in HT had significantly higher post-operative complication rate $(p<0.005)$. Of the 148 patients with HT, $31.6 \%$ (77) had hypocalcaemia while $68.4 \%$ (167) with benign goitres had hypocalcaemia. In addition, $51.8 \%$ of the patients in the HT group were later diagnosed with malignancy; there were no malignancies present in the group $B$.

Conclusion

Though patients with HT suffer higher rate of complications after thyroidectomy when compared to benign goitres, careful pre-operative planning and risk counselling of patients with improved surgical techniques helps to minimize postoperative morbidity.

$\underline{\text { Keywords }}$

Hashimoto Thyroiditis; Goitre, Benign; Hemithyroidectomy; Hypocalcaemia

$\mathrm{T}$ Thyroid disease is a frequently encountered endocrine disease in India. ${ }^{1}$ Its incidence is very high. Thyroid diseases can be divided into benign and malignant types. Hashimoto's thyroiditis (HT) is one of the most common autoimmune diseases characterised by the production of antithyroid antibodies such as anti-thyroperoxidase (TPO) and anti-thyroglobulin

1 - Department of ENT, Otorhinolaryngology, Father

Muller Medical College, Mangalore

\section{Corresponding author:}

Dr Anita Aramani

email: anuaramani@yahoo.com antibodies (TG-Ab), that destroys the thyroid tissue leading to a decrease in the normal thyroid function. ${ }^{2}$ Patients with HT who develop hypothyroidism are treated with thyroid replacement and surgery is rarely necessary. ${ }^{3}$ Thyroidectomy in HT is not generally recommended because the inflammation that surrounds the gland can make the resection difficult, with excessive traction on the recurrent laryngeal nerve and injury to the parathyroid glands. Indications for surgery in HT include significant compressive symptoms, suspicion of malignancy and persistent symptoms associated with the disease. ${ }^{2,3}$ Following thyroidectomy early, intermediate and late complications can occur and among them 
injury to recurrent laryngeal nerve, parathyroid gland damage, and post-operative haematoma are considered complications of technique. ${ }^{4,5}$ Some thyroidectomies in conditions like hyperthyroidism, goitre and thyroiditis are more difficult to tackle and the degree of difficulty varies widely, and it is not easy to predict the level of difficulty preoperatively. ${ }^{6}$ In HT patients the incidence of complications could be high due to adherence of the thyroid gland to its anatomical surroundings. The present study was undertaken to compare the postoperative complication rates after thyroidectomy in HT patients versus benign goitres.

\section{Materials and Methods}

In this study we retrospectively reviewed the data from patients who underwent thyroidectomy for benign goitres between January 2013 and December 2017 in a tertiary care Hospital. The Hospital is a teaching hospital affiliated with a Medical College. Institutional Ethics Committee clearance (IEC/CCM/447/2018) was obtained for waiver of consent as there was no contact between the researchers and patients, this being a retrospective study.

Patients who underwent total thyroidectomy were divided into two groups. Group A included patients confirmed to have Hashimoto thyroiditis (HT) on fine needle aspiration cytology and ultrasonography. Group $B$ included patients who underwent thyroidectomy for other benign thyroid disorders. Patient data was searched for symptoms like compression in the neck, voice changes, and hormone imbalance. Other parameters assessed were pre-operative investigations, indications for surgery, type of surgery, complications and prevalence of malignancy. From the total of 578 patients included in the study, 148 patients confirmed to have HT on fine needle aspiration cytology and ultrasonography formed Group A. We compared this group with the remaining 430 patients who underwent thyroidectomy for other benign thyroid disorders (Group B). Only the patients who underwent total thyroidectomy were included in the study. Patients undergoing concomitant neck dissection, parathyroidectomy, and revision thyroidectomies were excluded. Patients having a preoperative diagnosis of thyroid cancer were also excluded. Vocal cord function was checked by laryngoscopy by an Otorhinolayngologist before surgery in all patients. A diagnosis of HT was done based on the presence of lymphocytic infiltration of stroma, Hurthle cell change of follicular epithelium on fine needle aspiration cytology in addition to serum thyroid hormone levels and ultrasonographic findings. A final histopathological evaluation was also considered in these patients. Thyroperoxidase (TPO) antibodies and antithyroglobulin antibodies were not measured in all patients; hence they were not taken into consideration. Post-operatively patients were evaluated for complications like hoarseness of voice due to recurrent laryngeal nerve palsy, hypocalcaemia, reexploration, and infection. The results were expressed in percentages, proportions. The continuous data was presented as means and standard deviation. The student $t$ test was applied to find out whether significant mean differences existed in ages of the patients who underwent thyroidectomy for Benign Thyroid disease and Hashimoto thyroiditis. The Appropriate tests of association like Chi square test were used to find out association of post-operative complications with the indications for thyroidectomy. The data was analysed using SPSS 23 and p-value $<0.05$ was considered as statistically significant.

\section{Results}

There was no statistical significant difference in the age of patients between the two groups. The patients in our series ranged from 13-70 years with mean age of $45+10.5$ years for group A and $46+11.7$ years for group B. In both groups females were commonly affected. (Table I) While looking at postoperative complications we focussed mainly on hypoparathyroidism and hoarseness of voice due to recurrent laryngeal nerve injury. Of the 148 patients with HT, 31.6\% (77) had hypocalcaemia; and $68.4 \%$ (167) with benign goitres had hypocalcaemia. (Table II) A range of 8.2-10 mg/ $\mathrm{dl}$ of serum calcium was considered normal and less than 8.2 was considered as hypocalcaemia. The lowest recorded value was $5.4 \mathrm{mg} / \mathrm{dl}$ in benign goitres and $6.8 \mathrm{mg} / \mathrm{dl}$ in HT. Furthermore, one patient had vocal cord palsy in HT, whereas 3 patients had vocal cord palsy in 
Table I: Demographics of HT patients compared to Benign Goitre patients

\begin{tabular}{|c|c|c|c|c|l|}
\hline \multicolumn{2}{c}{} & \multicolumn{2}{c}{ BENIGN } & \multicolumn{2}{c|}{ HASHIMOTO'S } \\
\cline { 3 - 7 } & & COUNT & N \% & COUNT & N \% \\
\hline \multirow{3}{*}{ AgeGr } & $<=45$ & 208 & $48.40 \%$ & 67 & $45.30 \%$ \\
\cline { 2 - 6 } & $>45$ & 222 & $51.60 \%$ & 81 & $54.70 \%$ \\
\hline \multirow{3}{*}{ Sex } & Male & 59 & $13.70 \%$ & 7 & $4.70 \%$ \\
\cline { 2 - 6 } & Female & 371 & $86.30 \%$ & 141 & $\mathbf{9 5 . 3 0 \%}$ \\
\hline
\end{tabular}

benign goitres. One patient in Group B had developed haemorrhage and needed neck exploration. Hence when the complication rate was compared between the groups the p-value was found to be 0.005 which was statistically significant stating patients with HT had higher rate of complications. In addition, 48.1\% (51) HT patients were diagnosed to have papillary carcinoma, $0.9 \%$ (1) patient had medullary carcinoma, and 2.8\% (3) patients had follicular carcinoma on final histopathological evaluation. (Table III)

\section{Discussion}

Total thyroidectomy is an effective treatment for benign as well as malignant disease.

Articles by Kocher, Halsted, Lahey, Crile and Riddle have provided surgeons with principles that have significantly reduced the morbidity associated with thyroidectomy. ${ }^{5,7}$ Patients with HT are managed either conservatively by medical management or surgically.
Treatment generally depends on the presenting symptoms and thyroidectomy is considered for patients with symptoms like dysphagia, dyspnoea resulting from compression of oesophagus and trachea. ${ }^{8}$ In addition, thyroidectomy can be considered when there are symptoms like pressure or pain in the neck with discomfort, hoarseness of voice and fluctuation between symptoms of hypothyroidism and hyperthyroidism and suspicion of malignancy. ${ }^{9}$ However, thyroidectomy in HT is not usually recommended because the surgical resection is more difficult due to the dense inflammatory process that surrounds the gland, making complications like hypoparathyroidism due to trauma or disruption of blood supply to parathyroid glands and recurrent laryngeal nerve injury due to adherence of the gland, commoner. ${ }^{2,10,11}$

In a study by Catherine et al. ${ }^{2}$ patients with HT were compared with those patients without HT with regard to outcomes. They reported that patients with HT had a higher rate of complications like transient $(9.6 \%)$

Table II: Hypocalcemia in HT patients compared to Benign Goitre patients

\begin{tabular}{|c|c|c|c|}
\hline \multirow{2}{*}{ NORMAL } & HYPOCALCEMIA & TOTAL \\
\cline { 2 - 4 } & 208 & $48.40 \%$ & 67 \\
\cline { 2 - 4 } & 222 & $51.60 \%$ & 81 \\
\hline \multirow{2}{*}{ Sex } & 59 & $13.70 \%$ & 7 \\
\cline { 2 - 4 } & 371 & $\mathbf{8 6 . 3 0 \%}$ & 141 \\
\hline \multirow{2}{*}{ Total } & 334 & $\mathbf{2 4 4}$ & $\mathbf{5 7 8}$ \\
\cline { 2 - 4 } & $100.00 \%$ & $100.00 \%$ & $100.00 \%$ \\
\hline
\end{tabular}


Table III: Incidence of malignancy in the histopathology report in HT patients compared to Benign Goitre patients

\begin{tabular}{|c|c|c|c|c|c|}
\hline & $\begin{array}{c}\text { BENIGN } \\
\text { THYROID } \\
\text { DISEASE }\end{array}$ & $\begin{array}{c}\text { PAPILLARY } \\
\text { CARCINOMA }\end{array}$ & $\begin{array}{c}\text { MEDULLARY } \\
\text { CARCINOMA }\end{array}$ & $\begin{array}{c}\text { FOLLICULAR } \\
\text { CARCINOMA }\end{array}$ & TOTAL \\
\hline \multirow{2}{*}{ Benign } & 430 & 0 & 0 & 0 & 430 \\
\cline { 2 - 7 } & $100 \%$ & $0 \%$ & $0 \%$ & $0 \%$ & $100 \%$ \\
\hline \multirow{2}{*}{ Hashimotos's } & 51 & 51 & 1 & 3 & 106 \\
\cline { 2 - 6 } & $48.10 \%$ & $48.10 \%$ & $0.90 \%$ & $2.80 \%$ & $100 \%$ \\
\hline
\end{tabular}

and permanent $(2.6 \%)$ hypoparathyroidism, permanent hoarseness $(1.3 \%)$. There was no significant difference between two groups in the rate of malignancy. Of the 133 patients, $28(21 \%)$ patients experienced postoperative complications; including three patients who had multiple complications and $2 \%$ of patients did not experience relief of preoperative symptoms after surgery. They also concluded that the presence of malignancy was not responsible for the difference in postoperative complication rates between HT and nonHT patients.

Mok et al. ${ }^{6}$ used thyroidectomy difficulty scale to score the difficulty of thyroid operations. They compared difficult versus non-difficult thyroids. Their study showed that hyperthyroidism, elevated preoperative thyroglobulin, antithyroglobulin antibodies and Grave's ophthalmopathy were associated with a more difficult thyroid surgery and more complications. Of the 189 patients, $40 \quad(21.2 \%)$ experienced postoperative complications like hoarseness of voice (6.9\%), hypoparathyroidism $(23.3 \%)$. However, these measures of difficulty may not apply to all surgeons, especially those with lower thyroidectomy volumes. Manus et al. ${ }^{9}$ performed a study in which they identified patients diagnosed with HT with significant preoperative symptoms who were evaluated for improvement or relief of symptoms postoperatively. The study showed compression as the most common symptom at a frequency of $63 \%$; and $93 \%$ of the patients experienced post-operative relief. Though there were some patients with transient complications, which resolved within 6 months, overwhelming majority of symptomatic patients experienced benefit from thyroidectomy.

Shih et al. ${ }^{12}$ retrospectively evaluated HT patients who underwent thyroidectomy for indications like thyroid cancers, benign thyroid nodules and relief of local symptoms. Of the 474 patients, $53 \%$ had thyroid cancer at final histopathological examination. $32.1 \%$ had transient postoperative hypocalcaemia, $0.4 \%$ had transient recurrent laryngeal nerve palsy, and $0.8 \%$ had a postoperative neck haematoma. No death or permanent surgical complications were seen. Hence, they concluded that thyroidectomy can be performed in patients with HT with a low risk of permanent surgical complications. In addition, they also found that cancer was also common in patients with HT even when not suspected preoperatively.

The purpose of the present study was to compare the postoperative complications among HT and benign goitres after thyroidectomy. Majority of the patients were females in both the groups. As studies ${ }^{2,6,12}$ show that complication rate among HT is higher, our study too showed a statistically significant difference ( $\mathrm{p}$ value 0.005) in HT patients having higher rate of complications especially hypocalcaemia.

The findings of the present study suggest that thyroidectomy in HT is warranted in view of the hidden malignancy which may not be picked by fine needle aspiration cytology. Shih et al ${ }^{12}$ reported that $7(28 \%)$ of 25 patients who underwent surgery for thyroiditis alone had an incidentally discovered cancer, suggesting that cancer is common in patients with HT even when not suspected preoperatively. Our study too had a higher positivity for malignancy in the final histopathology which was not suspected preoperatively. $48.1 \%$ (51) patients had an association with papillary carcinoma. The present study had some limitations. This being a chart-based study, we were unable to know whether 
the complications were transient or permanent. Not all patients had details of anti-TPO and anti-thyroglobulin antibodies as some were evaluated on outpatient basis and hence details were missing in the charts. Risk of complications depends upon extent of thyroid gland resection, which can be minimized with experience and appropriate technique. Time taken for these surgeries may be a little longer and needs planning. Though thyroidectomy is not mandatory in HT, it must be considered in specific cases.

\section{Conclusion}

In the present study, patients with HT undergoing thyroidectomy had a significant higher rate of postoperative complications compared to benign goitres. However, though thyroidectomy is technically more demanding in HT a careful preoperative planning with patient counselling regarding risks of surgery needs to be considered. Another advantage of surgery would also be reducing long-term risk of malignancy. Hence it is evident that thyroidectomy in $\mathrm{HT}$ requires an experienced and skilled surgeon with improved surgical techniques to minimize postoperative mortality and morbidity.

\section{References}

1. Padur AA, Kumar N, Guru A, Badagabettu SN, Shanthakumar SR, Virupakshamurthy MB, et al. Safety and effectiveness of total thyroidectomy and its comparison with subtotal thyroidectomy and other thyroid surgeries: a systematic review. J Thyroid Res. 2016; 2016:7594615. doi: 10.1155/2016/7594615

2. McManus C, Luo J, Sippel R, Chen H. Is thyroidectomy in patients with Hashimoto's thyroiditis more risky? J Surg Res. 2012;178: 529-2

3. Thomas CG Jr, Rutledge RG. Surgical intervention in chronic (Hashimoto's) thyroiditis. Ann Surg. 1981;193:769-6

4. Wormer BA, McHenry CR. Hashimoto's thyroiditis: Outcome of surgical resection for

5. patients with thyromegaly and compressive symptoms. Am J Surg. 2011;201:416-9

6. Azim MA, Karim AK,Habib MA,Mondal MS, Anowar MA.Thyroid Surgery: Complications and Techniques. Dinajpur Med Col J. 2017;10:1-9

7. Mok VM, Oltmann SC, Chen H, Sippel RS, Schneider DF. Identifying predictors of a difficult thyroidectomy. J Surg Res. 2014; $190: 157-3$

8. Harness JK, Fung L, Thompson NW, Burney RE, McLeod MK. Total thyroidectomy: complications and technique. World J Surg. 1986 ; 10:781-6

9. Tajiri J. Radioactive iodine therapy for goitrous Hashimoto's thyroiditis. J Clin Endocrinol Metab. 2006; 91:4497-500

10. McManus C, Luo J, Sippel R, Chen H. Should patients with symptomatic Hashimoto's thyroiditis pursue surgery? J Surg Res. 2011;170:52-5

11. Youngwirth L, Benavidez J, Sippel R, Chen H. Parathyroid hormone deficiency after total thyroidectomy: incidence and time. J Surg Res. 2010; 163:69-1

12. Glinoer D, Andry G, Chantrain G, Samil N. Clinical aspects of early and late hypocalcaemia after thyroid surgery. Eur J Surg Oncol. 2000; 26:571-7

13. Shih ML, Lee JA, Hsieh CB, Yu JC, Liu HD, Kebebew E, et al. Thyroidectomy for Hashimoto's thyroiditis: complications and associated cancers. Thyroid 2008;18:729-4. 